\title{
THEMIS observations of plasma transport via eddy diffusion
}

\author{
T. Izutsu ${ }^{1}$ and M. Fujimoto ${ }^{2}$ \\ ${ }^{1}$ Department of Earth and Planetary Science, The University of Tokyo, Tokyo, Japan \\ ${ }^{2}$ Institute of Space and Astronautical Science, Japan Aerospace Exploration Agency, Kanagawa, Japan
}

Correspondence to: T. Izutsu (izutsu@stp.isas.jaxa.jp)

Received: 15 October 2012 - Revised: 3 December 2012 - Accepted: 4 December 2012 - Published: 17 December 2012

\begin{abstract}
We provide an event study of THEMIS observations of the low-latitude boundary layer in the dayside magnetosphere. Simultaneous multipoint observations obtained on 5 December 2008 show that the magnetosheathlike plasma in the low-latitude boundary layer is transferred earthward from the magnetopause. This earthward transport is accompanied by decrease in the density and fluctuating bulk flow. We calculate the eddy diffusion coefficients, which can be estimated from the observed velocity data, and found that the numbers are in good quantitative agreement with the spatial and time scales of the observed earthward transport signatures. It is shown that other possible plasma transport processes such as convection or diffusion induced by plasma wave turbulence are inconsistent with the observations. Our study strongly suggests that the observed transport is due to diffusive transport via turbulent eddy motions as is the case of an ordinary (Navier-Stokes) fluid.
\end{abstract}

Keywords. Magnetospheric physics (Magnetopause, cusp, and boundary layers) - Space plasma physics (Transport processes; Turbulence)

\section{Introduction}

Understanding the transport mechanism of material particles (e.g., constituent molecule or pollutant) in various fluid media (e.g., atmosphere or ocean) is important in many industrial and geophysical problems. It is well known that turbulence can transfer those particles more efficiently than molecular diffusion. This mode of transport is called eddy diffusion or turbulent diffusion (e.g., Hinze, 1975).

For collisionless space plasmas, the concept of eddy diffusion involves multi-scale physics (e.g., Borovsky and Gary, 2009) and is not necessarily a straightforward one. Nevertheless, eddy diffusion has been considered to play impor- tant roles, for example, in the control of the structure of the low-latitude boundary layer (LLBL) (Antonova, 2005), in the transfer of magnetosheath plasma into the midnight neutral sheet from the plasma sheet boundary layer (Borovsky et al., 1998) or from the magnetospheric flanks (Wang et al., 2010), or in making the plasma sheet in the magnetostatic equilibrium whose collapse may be related to the substorm onset (Antonova and Ovchinnikov, 1999).

To date, there has not been an in-depth study to prove that eddy diffusion does indeed operate in space plasmas. In previous studies, discussions on eddy diffusion in the magnetosphere have been based on coarse-grained datasets that have a time scale of hours and a spatial scale larger than $1 R_{\mathrm{E}}$. The datasets of these characteristics have been subjected to statistical studies (Borovsky et al., 1998; Wang et al., 2010) or to single-spacecraft studies (Borovsky et al., 1998; Wing et al., 2006). In contrast, in this paper, we will try to put forward a more solid argument for plasma eddy diffusion on the basis of simultaneous multipoint observations. The dataset has a time scale of ten minutes and a spatial scale less than $1 R_{\mathrm{E}}$, from which we can make a more solid test regarding whether the observed signatures match the expectation from eddy diffusion framework.

\section{Event overview}

We searched for simultaneous multipoint observations of magnetosheath-like plasma existing in the dayside magnetosphere. Such cold-dense plasma would be a marker of a plasma transport process earthward from the dayside magnetopause. THEMIS (Angelopoulos, 2008) data obtained in 2007 and 2008 have been inspected. We found one event in which cold-dense plasma was observed at more than two spacecraft and the density profiles at first sight are suggestive of diffusive transport. 

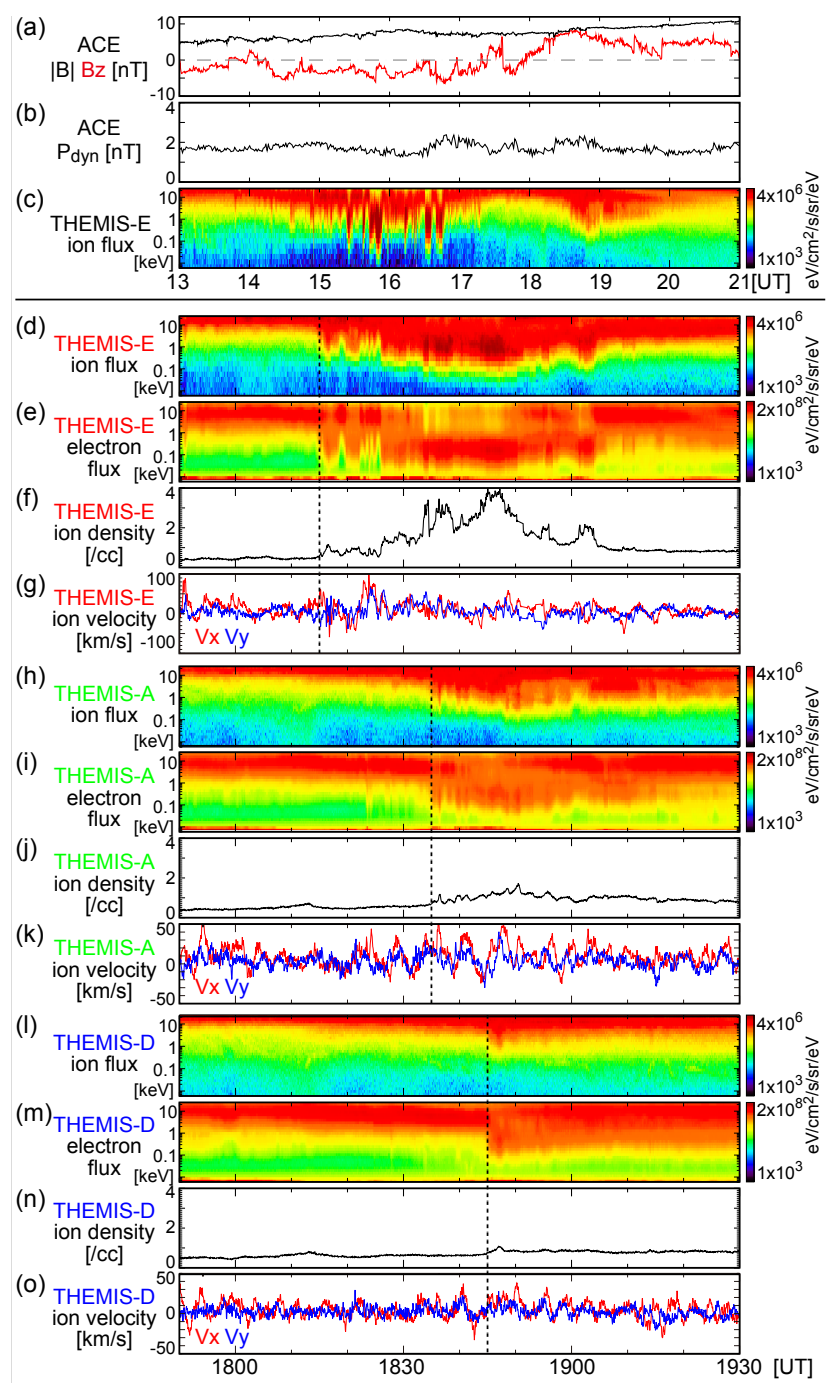

Fig. 1. Time series plots of measurements by ACE (shifted by 55 min) and THEMIS on 5 December 2008; (a) ACE IMF, (b) ACE solar wind dynamic pressure, and (c) THEMIS-E ion energy spectrogram during 13:00-21:00 UT; (d) ion energy spectrogram, (e) electron energy spectrogram, (f) ion density, and (g) ion velocity measured by THEMIS-E during 17:50-19:30 UT; the same measurements as in (d)-(g) but from THA and THD are plotted in (h)-(k) and (l)-(o), respectively. Vertical dashed-lines in (d)-(o) indicate the detection of the inner edge of the LLBL by each spacecraft.

Figure 1 shows an overview of the event. Data from the ion and electron plasma instruments (McFadden et al., 2008) onboard THEMIS-E (THE), THEMIS-A (THA), and THEMIS-D (THD) are used in this study. The three spacecraft were in the dawn-morning sector of the magnetosphere and were close to the magnetopause. All three spacecraft detected plasma in the LLBL which is characterized by the coexistence of cold magnetosheath-like and hot magnetospheric plasmas (Fig. 1c, d, e, h, i, l and m) and by bi- directional field-aligned fluxes of $\sim 100 \mathrm{eV}$ electrons (not shown).

Figure 1c shows the data obtained by THE during the entire $8 \mathrm{~h}$ interval. The position of THE was $X_{\mathrm{GSM}}=0.17 \sim 4.3 R_{\mathrm{E}}, \quad Y_{\mathrm{GSM}}=-11 \sim-8.0 R_{\mathrm{E}}, \quad$ and $Z_{\mathrm{GSM}}=0.74 \sim-0.41 R_{\mathrm{E}}$. The LLBL was encountered intermittently in 14:30-17:30 UT when the IMF was directed southward (Fig. 1a). The LLBL feature was gradually enhanced from 18:15 UT, 20 min after the northward turning of the IMF. Thickening of LLBL under northward IMF has been noted by various studies (e.g., Mitchell et al., 1987; Øieroset et al., 2008) and this particular event seems to follow the well-known rule.

Here we focus on the latter LLBL observations (Fig. 1do). The inner edge of the LLBL plasma was observed sequentially by THE at 18:15 UT, by THA at 18:35 UT and by THD at 18:45 UT. The positions of each spacecraft upon respective encounter with the LLBL inner-edge are shown by cross marks in Fig. 2. The sequence of the LLBL detections by THE, THA, and THD indicates that the LLBL plasma is transferred earthward.

The observed transport would not be due to convective flow. Constant solar wind dynamic pressure (Fig. 1b) indicates that compression of the magnetosphere would not have occurred, and ion bulk flow detected by each THEMIS spacecraft is not streaming in a specific direction but is highly fluctuating (Fig. 1g, $\mathrm{k}$ and o). Decrease in the peak density of ions with approaching to the Earth (Fig. 1f, $\mathrm{j}$ and $\mathrm{n}$ ) is inconsistent with what is expected for convection or magnetospheric compression. Instead the fluctuating nature and the density decrease imply operation of diffusive transport via turbulence. Below, we investigate the data obtained by the three spacecraft from the viewpoint of eddy diffusion.

\section{Analysis and results}

Here, we calculate diffusion coefficients $D=d\left\langle x^{2}\right\rangle /(2 d t)$ in two independent ways and compare them with each other: one is to obtain the diffusion coefficient from the timing of two point observations of the LLBL plasma and the other is to estimate the eddy diffusion coefficient from observed velocity data.

For the current purpose, the normal angle of the LLBL plasma front is needed. It would be reasonable to consider that the LLBL plasma is broadened in the direction along the LLBL normal. The angle to define the normal, which is depicted as $\theta$ in Fig. 2, can be estimated by so-called timing analysis (Schwartz, 1998). Since the normal vector of the LLBL would lie in X-Y GSM plane, data from three points can determine $\theta$. Because the timing analysis cannot be applied to a diffusive transport event in which the constant velocity assumption is not valid, we apply the method to the intermittent LLBL encounters at 16:30 UT when the inner edge of the LLBL is clearly detectable by all the three spacecraft. 


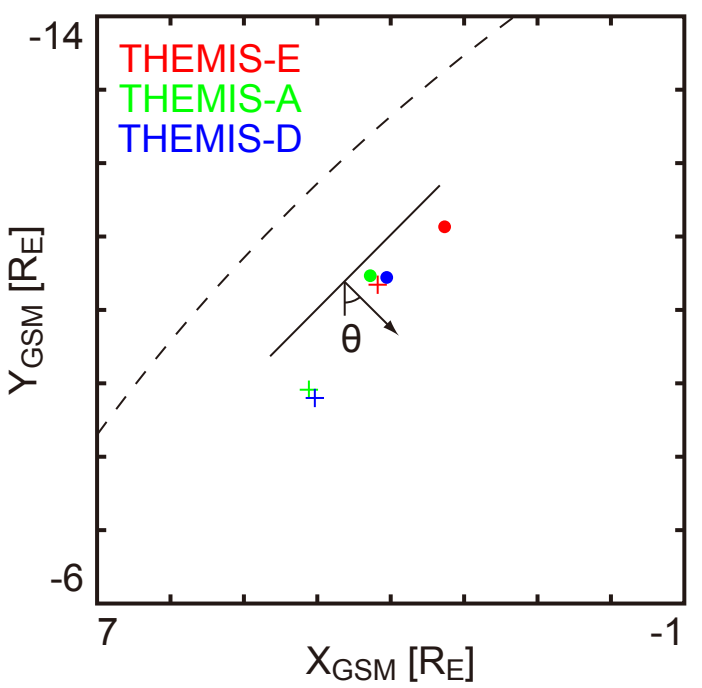

Fig. 2. Depicted by circles are the positions of the inner edge of the LLBL detected by THA at 16:30:32 UT (green), THD at 16:30:38 UT (blue), and THE at 16:31:20 UT (red) that are used to determined the LLBL front normal direction. Crosses show the positions of the inner edge of the LLBL detected by THE at 18:15 UT (red), THA at 18:35 UT (green), and THD at 18:45 UT (blue) that are used to determine the characteristics of eddy diffusion transport. Possible magnetopause position is shown by dashed-line as a reference.

We obtained $\theta=39.2-45.2^{\circ}$ from the LLBL detections by THA at 16:30:32 UT, by THD at 16:30:38 UT, and by THE at 16:31:20-16:31:26 UT, respectively. The positions of each spacecraft at these times are shown by circles in Fig. 2. While the results shown in the following sections are for $\theta=42^{\circ}$, we obtained the same conclusion for the range of $\theta$ indicated above.

Now we evaluate the diffusion coefficient from the spatial and time scales of the LLBL observations at two-point. We suppose that the relative distance (transferred distance) between the first detection location of the LLBL inner edge by one spacecraft and the subsequent detection location by the other spacecraft is determined by the root-mean-square displacement of particles subject to diffusion. If the diffusion coefficient is constant in time, the transferred distance can be written as $\Delta x=\sqrt{2 D \Delta t}$, where $\Delta t$ is the elapsed time. Using the time and position of the first LLBL detection by spacecraft 1 ( $\mathrm{SC} 1$ ) and those of the subsequent detection by $\mathrm{SC} 2$, the diffusion coefficient can be estimated by $D_{\mathrm{SC}}(\mathrm{SC} 1-\mathrm{SC} 2)=\Delta x_{\mathrm{SC}}(\mathrm{SC} 1-\mathrm{SC} 2)^{2} /(2 \Delta t)$, where $\Delta x_{\mathrm{SC}}(\mathrm{SC} 1-\mathrm{SC} 2)=x_{\mathrm{SC} 2}-x_{\mathrm{SC} 1}$ along the normal direction and $\Delta t=t_{\mathrm{SC} 2}-t_{\mathrm{SC} 1}$. Note that we implicitly assumed that the distance $\Delta x$ and the duration $\Delta t$ is much larger than the spatial and time scales of elementary processes that build-up the diffusive transport. We calculate two $D_{\mathrm{SC}}$ 's from two pairs of two-point observations: $D_{\mathrm{SC}}$ (E-A) from the LLBL detections by THE at 18:15 UT and by

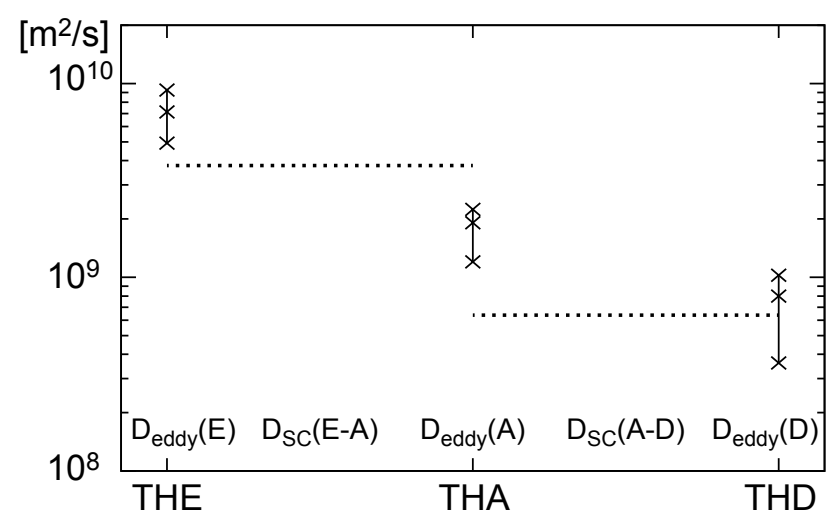

Fig. 3. Dotted: $D_{\mathrm{SC}}$, the diffusion coefficient estimated from the two-point observations. Solid: $D_{\text {eddy }}$, the eddy diffusion coefficient calculated from observed velocity data at each spacecraft position. Shown are the minimum, median, and maximum values. See details in the text.

THA at 18:35 UT, in which $\Delta x_{\mathrm{SC}}(\mathrm{E}-\mathrm{A})=3000 \mathrm{~km}$, and $D_{\mathrm{SC}}$ (A-D) from those by THA at 18:35 UT and by THD at 18:45 UT, in which $\Delta x_{\mathrm{SC}}(\mathrm{A}-\mathrm{D})=870 \mathrm{~km}$. Shown in Fig. 3 are the calculated values: $D_{\mathrm{SC}}(\mathrm{E}-\mathrm{A})=3.8 \times 10^{9} \mathrm{~m}^{2} \mathrm{~s}^{-1}$ and $D_{\mathrm{SC}}(\mathrm{A}-\mathrm{D})=6.4 \times 10^{8} \mathrm{~m}^{2} \mathrm{~s}^{-1}$. The decrease in $D_{\mathrm{SC}}$ with decreasing distance to the Earth implies that the diffusion process that broadens the LLBL becomes weaker as its inneredge propagates earthward.

Next we evaluate the eddy diffusion coefficient. Previous studies on eddy diffusion in the magnetosphere (Borovsky et al., 1997, 1998; Wang et al., 2010; Stepanova et al., 2011) have adopted the following formula: $D_{\text {eddy }}=(1 / 2) u_{\mathrm{rms}}^{2} \tau_{\mathrm{ac}}$, where $u_{\mathrm{rms}}$ is a root-mean-square of the velocity and $\tau_{\mathrm{ac}}$ is an auto-correlation time. The auto-correlation time was determined as the best fit to the natural logarithm of an autocorrelation function $R(\tau)=\exp \left(-\tau / \tau_{\mathrm{ac}}\right) ; R(\tau)$ is calculated for a given time interval including $N$ data points by $R(\tau)=$ $\sum_{i=0}^{N}\left(u(i)-u_{\mathrm{ave}}\right)\left(u(i+\tau)-u_{\mathrm{ave}}\right) /\left(N u_{\mathrm{rms}}^{2}\right)$ where $u$ is observed velocity in the direction of the LLBL normal (specified by $\theta$ ), $u_{\mathrm{ave}}=\sum_{i=0}^{N} u(i) / N$ and $u_{\mathrm{rms}}=$ $\sqrt{\sum_{i=0}^{N}\left(u(i)-u_{\mathrm{ave}}\right)^{2} / N}$. We should point out that these are based on the knowledge of an ordinary (Navier-Stokes) fluid (Borovsky et al., 1998) and that the accurate formula of $D_{\text {eddy }}$ for magnetized, collisionless space plasmas is yet unknown and should be studied in the future.

Three $D_{\text {eddy }}$ 's are estimated: $D_{\text {eddy }}(E)$ from the velocity data of THE during 18:15-18:30 UT, $D_{\text {eddy }}(A)$ from data of THA during 18:35-18:50 UT, and $D_{\text {eddy }}(D)$ from data of THD during 18:45-19:00 UT. For each interval, we calculate eight values of $D_{\text {eddy }}$ by setting eight 8 -min subintervals with the start time shifted by $1 \mathrm{~min}$, and select the minimum, median, and maximum values among them. The calculated values are shown in Fig. 3: the median values are $D_{\text {eddy }}(E)=7.1 \times 10^{9} \mathrm{~m}^{2} \mathrm{~s}^{-1}, D_{\text {eddy }}(A)=1.9 \times 10^{9} \mathrm{~m}^{2} \mathrm{~s}^{-1}$, 
and $D_{\text {eddy }}(D)=8.0 \times 10^{8} \mathrm{~m}^{2} \mathrm{~s}^{-1}$ which are, respectively, composed of $\left(u_{\mathrm{rms}}, \tau_{\mathrm{ac}}\right)=\left(24 \mathrm{~km} \mathrm{~s}^{-1}, 24 \mathrm{~s}\right),\left(11 \mathrm{~km} \mathrm{~s}^{-1}\right.$, $33 \mathrm{~s})$, and $\left(8 \mathrm{~km} \mathrm{~s}^{-1}, 25 \mathrm{~s}\right)$. Note that the characteristic spatial scales of eddies, $l_{\text {eddy }} \sim u_{\mathrm{rms}} \tau_{a c}$ (Borovsky et al., 1997), are estimated to be $590 \mathrm{~km}$ at THE, $360 \mathrm{~km}$ at THA, and $200 \mathrm{~km}$ at THD, respectively, which are sufficiently larger than gyroradii of the transferred particles (e.g., a proton with energy of $0.1-1 \mathrm{keV}$ has gyroradius of $30-80 \mathrm{~km}$ in our event). The values of $\tau_{\mathrm{ac}}$ is much smaller than $\Delta t$, namely, an eddy lifetime is much shorter than the time lags between consecutive LLBL encounters, which is consistent with what is assumed in obtaining $D_{\mathrm{SC}}$.

In Fig. 3, we found that $D_{\text {eddy }}$ becomes smaller for a spacecraft nearer to the Earth, as is the case for $D_{\mathrm{SC}}$. It is not only this qualitative consistency, but Fig. 3 also indicates the quantitative agreement between $D_{\mathrm{SC}}$ and $D_{\text {eddy }}$, if one notes that $D_{\mathrm{SC}}$ was derived under the assumption of the constant diffusion coefficient between each pair of spacecraft.

As another way of quantitative comparison, we calculate the transferred distance from the values of $D_{\text {eddy }}$ by taking into account the time dependence of the diffusion coefficient. Assuming that $D_{\text {eddy }}$ (SC1) decays to $D_{\text {eddy }}$ (SC2) according to an exponential temporal profile, the transferred distance of the LLBL inner edge from $t_{\mathrm{SC} 1}$ to $t_{\mathrm{SC} 2}$ can be evaluated from $\Delta x_{\text {eddy }}(\mathrm{SC} 1-\mathrm{SC} 2)=\left(\int_{t_{\mathrm{SC} 1}}^{t_{\mathrm{SC} 2}} 2 D_{\text {eddy }}(t) d t\right)^{1 / 2}$. The transferred distances are calculated to be $\Delta x_{\text {eddy }}(E \mathrm{~A})=$ $3100 \mathrm{~km}$ and $\Delta x_{\text {eddy }}(\mathrm{A}-\mathrm{D})=1200 \mathrm{~km}$ using the median values of $D_{\text {eddy }}$. These are in good quantitative agreement with the observed transferred distances $\Delta x_{\mathrm{SC}}(\mathrm{E}-\mathrm{A})=3000 \mathrm{~km}$ and $\Delta x_{\mathrm{SC}}(\mathrm{A}-\mathrm{D})=870 \mathrm{~km}$ with the errors between them being at most of the order of the typical size of an eddy. We also confirmed that the values of $D_{\text {eddy }}$ stay consistent with the observations when $\theta$ is varied in the range of 39.2-45.2 .

\section{Discussion}

Being different from an ordinary fluid, plasma transport can be obtained not only by fluid-like eddy turbulence but also by plasma wave turbulence. Wave-induced diffusion occurs via wave-particle interactions through Landau or cyclotron resonance (Treumann et al., 1995). A plausible candidate wave mode which can transfer a substantial amount of particles of lower energies is kinetic Alfvén wave (Hasegawa and Mima, 1978; Treumann et al., 1995). The lowest resonance energy, however, is $2.6 \mathrm{keV}(6.7 \mathrm{keV})$ at the inner edge of the LLBL observed by THE (THA). It shows that kinetic Alfvén wave turbulence can hardly explain the transport of the cold magnetosheath-like ions $(\sim 1 \mathrm{keV})$ detected in the LLBL.

As mentioned above, we found that convection, magnetospheric compression, and wave-induced diffusion are inconsistent with the observed signatures. Meanwhile the eddy diffusion coefficient obtained from the flow velocity data is consistent with the spatial and time scale characteristics seen by the multi-point observations. We conclude that the most likely plasma transport process for our event is eddy diffusion. This result validates the arguments in the previous studies (e.g., Borovsky et al., 1998; Antonova and Ovchinnikov, 1999; Antonova, 2005; Wang et al., 2010) which have a priori assumed the presence of plasma eddy diffusion. Especially the broadening of the LLBL due to eddy diffusion strongly supports the hypothesis by Antonova (2005) that the structure of the LLBL is a consequence of the balance between eddy diffusion and regular convective flow, which may result in the dependence of the LLBL thickness on the value and the direction of the IMF and on the dawn-dusk location.

It should be noted that the theory of eddy diffusion in collisionless space plasmas still has missing elements: the accurate expression for $D_{\text {eddy }}$ is not known and the microphysics of the transport process by which transported particles transfer from one eddy to another, in other words, how the frozen-in condition is violated upon the transfer from one vortex to another, remains open. Especially the latter makes us refrain from arguing that eddy diffusion would similarly cause transfer of magnetosheath plasma into the magnetosphere across the boundary of the magnetosphere: Sharp density contrast and current sheet set-up at the interface would make this issue even more complicated than the situation described here (transport within the magnetosphere). Dissipation of turbulent energy due to Landau damping or Bohm diffusion (Borovsky and Gary, 2009) may be related to the breakdown of the frozen-in condition and subsequent transport, which is beyond the scope of this paper. It would be worth noting for future works that the transferred distance in our event is at least five (ten) times the characteristic spatial scale of eddies observed at THE (THA), indicating that vortex transfer is necessary to enable the degree of LLBL broadening as observed.

The fact that the plasma can be transferred via eddy diffusion at least within the magnetosphere lets us re-consider diffusive plasma entry across the magnetopause. In order to form the LLBL of observed thickness via cross-field diffusion, the diffusion coefficient is required to exceed a certain value (Sonnerup, 1980). For example, the required diffusion coefficient $D=\left(\Delta_{\mathrm{MPCS}}+\Delta_{\mathrm{LLBL}}\right)^{2} /(2 T)$, where $\Delta_{\mathrm{MPCS}}$, $\Delta_{\mathrm{LLBL}}$, and $T$ are the thickness of the magnetopause current sheet (MPCS), that of the LLBL, and the transport time (the traveling time of the magnetosheath flow), respectively, is $\sim 5 \times 10^{9} \mathrm{~m}^{2} \mathrm{~s}^{-1}$ with typical values of $\Delta_{\text {MPCS }} \sim 500 \mathrm{~km}$, $\Delta_{\text {LLBL }} \sim 2000 \mathrm{~km}$, and $T=10 \mathrm{~min}$ (Phan and Paschmann, 1996; Bauer et al., 2001). Because eddy diffusion can well support the thickening of LLBL as observed, the diffusive process at the magnetopause, which is unknown at present, would only have to be in charge of transferring plasma across the magnetopause current sheet: hence, the required diffusion coefficient becomes $D=\Delta_{\text {MPCS }}^{2} /(2 T) \sim$ $2 \times 10^{8} \mathrm{~m}^{2} \mathrm{~s}^{-1}$, which is an order of magnitude smaller than the typical one. This rethinking may revive some of the 
candidates that have been discarded, such as lower hybrid drift instability (Treumann et al., 1995).

\section{Conclusion}

In this study, we analyzed an event in which LLBL plasma was encountered by three THEMIS spacecraft sequentially, which indicates the earthward broadening of the LLBL. The observed signatures such as the decrease in the peak density upon approaching to the Earth, fluctuating bulk motions without convective flow, constant solar wind dynamic pressure, and higher Alfvén velocity in the LLBL, are inconsistent with the plasma transport processes via convection, compression of the magnetosphere, or diffusion via plasma wave turbulence. We calculated eddy diffusion coefficients using observed velocity data, and found that these values are fairly consistent with the spatial and time scales derived from multipoint observations. Thus we conclude that the observed earthward transport of LLBL plasma would be due to diffusion induced via turbulent eddy motions therein. Thanks to simultaneous multipoint observations, we could verify that eddy diffusion indeed contributes to the plasma transport in the magnetosphere.

Acknowledgements. T. Izutsu is deeply grateful to H. Hasegawa and T. Nagai not only for insightful comments but also for teaching him how to address a problem. We acknowledge NASA contract NAS5-02099 and V. Angelopoulos for use of data from the THEMIS Mission. Specifically: C. W. Carlson and J. P. McFadden for use of ESA data. ACE data were provided via the CDAWeb.

Topical Editor R. Nakamura thanks M. Stepanova for her help in evaluating this paper.

\section{References}

Angelopoulos, V.: The THEMIS Mission, Space Sci. Rev., 141, 534, doi:10.1007/s11214-008-9336-1, 2008.

Antonova, E. E.: The structure of the magnetospheric boundary layers and the magnetospheric turbulence, Planet. Space Sci., 53, 161-168, doi:10.1016/j.pss.2004.09.041, 2005.

Antonova, E. E. and Ovchinnikov, I. L.: Magnetostatically equilibrated plasma sheet with developed medium-scale turbulence: Structure and implications for substorm dynamics, J. Geophys. Res., 104, 17289-17298, doi:10.1029/1999JA900141, 1999.

Bauer, T. M., Treumann, R. A., and Baumjohann, W.: Investigation of the outer and inner low-latitude boundary layers, Ann. Geophys., 19, 1065-1088, doi:10.5194/angeo-19-1065-2001, 2001.

Borovsky, J. E., Elphic, R. C., Funsten, H. O., and Thomsen, M. F.: The Earth's plasma sheet as a laboratory for flow turbulence in high- $\beta$ MHD, J. Plasma Physics, 57, 1-34, doi:10.1017/S0022377896005259, 1997.
Borovsky, J. E., Thomsen, M. F., and Elphic, R. C.: The driving of the plasma sheet by the solar wind, J. Geophys. Res., 103, 17617-17640, doi:10.1029/97JA02986, 1998.

Borovsky, J. E. and Gary, S. P.: On shear viscosity and the Reynolds number of magnetohydrodynamic turbulence in collisionless magnetized plasmas: Coulomb collisions, Landau damping, and Bohm diffusion, Phys. Plasmas, 16, 082307, doi:10.1063/1.3155134, 2009.

Hasegawa, A. and Mima, K.: Anomalous transport produced by kinetic Alfvéen wave turbulence, J. Geophys. Res., 83, 1117-1123, doi:10.1029/JA083iA03p01117, 1978.

Hinze, J. O.: Theory of Space Plasma Microinstabilities, McGrawHill, New York, 1975.

McFadden, J. P., Carlson, C. W., Larson, D., Ludlam, M., Abiad, R., Elliott, B., Turin, P., Marckwordt, M., and Angelopoulos, V.: The THEMIS ESA Plasma Instrument and Inflight Calibration, Space Sci. Rev., 141, 277-302, doi:10.1007/s11214-008-9440-2, 2008.

Mitchell, D. G., Kutchko, F., Williams, D. J., Eastman, T. E., and Frank, L. A.: An extended study of the low-latitude boundary layer on the dawn and dusk flanks of the magnetosphere, J. Geophys. Res., 92, 7394-7404, doi:10.1029/JA092iA07p07394, 1987

Øieroset, M., Phan, T. D., Angelopoulos, V., Eastwood, J. P., McFadden, J., Larson, D., Carlson, C. W., Glassmeier, K. H., Fujimoto, M., and Raeder, J.: THEMIS multi-spacecraft observations of magnetosheath plasma penetration deep into the dayside low-latitude magnetosphere for northward and strong By IMF, Geophys. Res. Lett., 35, L17S11, doi:10.1029/2008GL033661, 2008.

Phan, T. D. and Paschmann, G.: Low-latitude dayside magnetopause and boundary layer for high magnetic shear 1 . Structure and motion, J. Geophys. Res., 101, 7801-7816, doi:10.1029/95JA03752, 1996.

Schwartz, S. J.: Shock and Discontinuity Normals, Mach Numbers, and Related Parameters, ISSI Scientific Reports Series, 1, 249270, 1998.

Sonnerup, B. U. O.: Theory of the low-latitude boundary layer, J. Geophys. Res., 85, 2017-2026, doi:10.1029/JA085iA05p02017, 1980.

Stepanova, M., Pinto, V., Valdivia, J. A., and Antonova, E. E.: Spatial distribution of the eddy diffusion coefficients in the plasma sheet during quiet time and substorms from THEMIS satellite data, J. Geophys. Res., 116, A00I24, doi:10.1029/2010JA015887, 2011.

Treumann, R. A., Labelle, J., and Bauer, T. M.: Diffusion Processes: An Observational Perspective, p. 331, the American Geophysical Union, 1995.

Wang, C.-P., Lyons, L. R., Nagai, T., Weygand, J. M., and Lui, A. T. Y.: Evolution of plasma sheet particle content under different interplanetary magnetic field conditions, J. Geophys. Res., 115, A06210, doi:10.1029/2009JA015028, 2010.

Wing, S., Johnson, J. R., and Fujimoto, M.: Timescale for the formation of the cold-dense plasma sheet: A case study, Geophys. Res. Lett., 33, L23106, doi:10.1029/2006GL027110, 2006. 